\title{
BETA DIVERSITY OF BIRDS (Passeriformes, Linnaeus, 1758) IN SOUTHERN AMAZON
}

\section{DIVERSIDADE BETA DE PÁSSAROS (Passeriformes, Linnaeus, 1758) NA AMAZÔNIA MERIDIONAL}

\author{
Welvis Felipe Fernandes Castilheiro ${ }^{1 *}$ \\ Manoel do Santos-Filho ${ }^{2}$ \\ Robson Flores de Oliveira ${ }^{2}$ \\ 1 University of Lisbon - UL, Faculty of Sciences, Lisbon, Portugal \\ ${ }^{2}$ Mato Grosso State University - UNEMAT, Faculty of Biological Sciences, Mato Grosso, Brazil \\ ${ }^{*}$ Corresponding author: welvisfelipe@gmail.com
}

\begin{abstract}
The importance of estimating the biological diversity and understanding how ecological specialization of species changes with spatially-organized habitats in undeniable. High beta diversity between neighboring places means an elevated number of species living within small distances, which usually are adapted to local conditions and highly vulnerable to anthropogenic actions as deforestation and burning. We investigated beta diversity of birds from the order Passeriformes in Southern Amazon, within landscapes with a large heterogeneous vegetation cover (habitats with flooded forest, dry forest, and marsh palm) through sound, observational, and photographic censuses. We marked 126 points in equidistant transects. A total of 148 species of birds were identified, distributed in 27 families. We found that $97 \%$ of the species foraged in flooded forest, $77 \%$ in dry forest and $19 \%$ in marsh palms, and only $18 \%$ foraged in the three habitats. An ordination analyses revealed which species showed the strongest preference to each habitat. The analysis for the Global beta diversity showed that this value is high (Whittaker: 7.7405), and the same pattern was obtained with the measure of pairwise dissimilarity. An influence of spatial distance was clearly observed in the cluster analysis and confirmed with a partial Mantel analysis; however, this was not observed at points that coincided with the transition and substitution of species. The influence of spatial distance in the dissimilarity index (beta diversity) was significant (r: 0.0608, p: 0.0049). The assemblage of species in small local populations with high beta diversity may be at risk if deforestation, selective logging, and poaching continue. In summary, this study provides important information on specific habitats with high beta diversity that may be at risk of destabilization of its populations due to continuing environmental changes imposed by humans.
\end{abstract}

Keywords: Amazon birds; conservation; distance influence; high richness. 


\section{Resumo}

Estimar a diversidade biológica e entender as mudanças acerca da especialização ecológica das espécies entre habitats em uma organização espacial é importante. A alta diversidade beta reflete numa pequena distância entre locais, mas que contêm alta incorporação de espécies, que geralmente possuem grande sensibilidade e não estão adaptadas ao estreitamento causado pelo desmatamento ou a presença de queimadas por ações antrópicas. Investigou-se a diversidade beta de pássaros (Passeriformes) na Amazônia Meridional em paisagens com coberturas vegetais mais heterogêneas: habitats de floresta alagada (iguapó), floresta de terra firme e buritis através de censo de audições, observações e fotografias. Marcaram-se pontos em equidistantes transectos. Em todo o estudo foram amostrados 126 pontos. Identificaram-se 148 espécies de pássaros da ordem Passeriformes, distribuídas em 27 famílias. A classificação sobre os habitats de forrageamento resultou em $97 \%$ das espécies que forrageiam em iguapó, $77 \%$ em floresta de terra firme e 18,1\% em buritis, apenas $18 \%$ forragem nos três habitats. A análise de ordenação mostrou quais espécies estão mais relacionadas com cada habitat, a análise para a Beta diversidade Global certificou que a diversidade beta é alta (Whittaker: 7,7405). Como era esperado encontrou-se o mesmo padrão quando foi utilizada a medida de dissimilaridade por pares. Na análise de agrupamento, pode-se ver claramente a influência da distância espacial, mas em alguns pontos isto não ocorre. Estes pontos indicam o momento de transição e substituição de espécies. Na maioria dos casos, a influência da distância espacial é predominante e confirmou-se na análise de Mantel parcial. A influência da distância espacial entre o índice de dissimilaridade (diversidade beta) foi significante (r:0,0608, p:0,0049). Esta composição de espécies organizadas em pequenas populacões locais mas com alta diversidade beta não pode ser exposta ao desmatamento, corte seletivo de madeira e a caça furtiva como tem acontecido. Este estudo provê informações de habitats específicos de alta diversidade beta que estão correndo risco na estabilidade de suas populacões em face às mudanças ambientais contínuas nesta área de estudo.

Palavras-chave: conservação; influência da distância; pássaros da Amazônia; riqueza.

Received on: April, 12 $2^{\text {th }}, 2016$

Accepted on: October, $4^{\text {th }}, 2016$

\section{Introduction}

Ecologists have long been curious to understand how biological diversity changes with the environment, in particular species specialized in different ecologies in spatially-organized habitats $^{(1)}$. The smaller fraction of regional gamma diversity corresponds to the local alpha diversity and represents the number of species within small areas of similar and uniform habitats. Beta diversity represents the rate of species variation (turnover rate) between habitats ${ }^{(2)}$. Beta diversity frequently measures the substitution of species without considering the relative abundance ${ }^{(3)}$. However, the inclusion of the relative abundance or frequency index allows a more informative evaluation of species diversity, especially when it varies between habitats ${ }^{(4)}$. Studies that compare variation in the number of species between local habitats are necessary to determine patterns, particularly in places in the world that are enriched in bird species ${ }^{(5)}$. In these areas, deforestation 
and controlled burnings for agricultural expansion along with hunting pressure are decreasing species richness.

High beta diversity along an environmental gradient reflect the specialization of species in habitats. The quantification of this parameter can be used to design strategies for protection of bird diversity in these $\operatorname{areas}^{(6)}$. A high beta diversity within small distances reflects the presence of abundant species between neighboring habitats, which generally are locally specialized and highly susceptible to anthropogenic action ${ }^{(3)}$. The spatial variation of birds composition with types of habitats is rarely measured, but it has shown to be important to define the area needed to protect some species ${ }^{(3)}$. There are some studies on the changing of the composition of animal communities along altitudinal gradients, but beta diversity in tropical systems is particularly misunderstood ${ }^{(7,8)}$. According to these studies, a gradual change in composition occurs with altitude, although some results may have been affected by the sampling method ${ }^{(9)}$. Instead of using the total animal community, quantification of beta diversity using a specific taxonomic group, such as birds (order Passeriformes), may be easier in terms of research planning ${ }^{(10)}$. Despite the apparent capacity for dispersion, many factors seem to influence variation of bird diversity or delimit their geographic distribution, for example the presence of rivers ${ }^{(4)}$. Habitats created by rivers, such as floodplain forest, flooded habitats, and habitats with fluctuations in vegetation, present about $15 \%$ regional birds, suggesting that watercourses may have had a relevant role in the origin of different bird species in the current region of Southern Amazon. The present study investigated the change in the composition of bird species of the order Passeriformes along gradients in the Alto-Guaporé region, Southern Amazon, a region that presents an elevated richness of birds and has a constant substitution of species due to a high diversity of environments. We aimed to answer two questions: Are spatially closed habitats more similar in species composition (beta diversity)? Are there differences in species richness among the type of sampled habitats (flooded forest, dry forest, and marsh palm with palm tree fruits)? We used the observed patterns of variation in species composition obtained within a bird community and explored possible implications for conservation planning.

\section{Methods}

This study was performed from December 2011 to September 2012, in three large localities (A,B,C) in the region of Alto-Guaporé in Southern Amazon, a region characterized by a heterogeneous vegetation cover (180879.94S, 8331318.14W between 780708.00S, 8507364W; see Fig.1) 


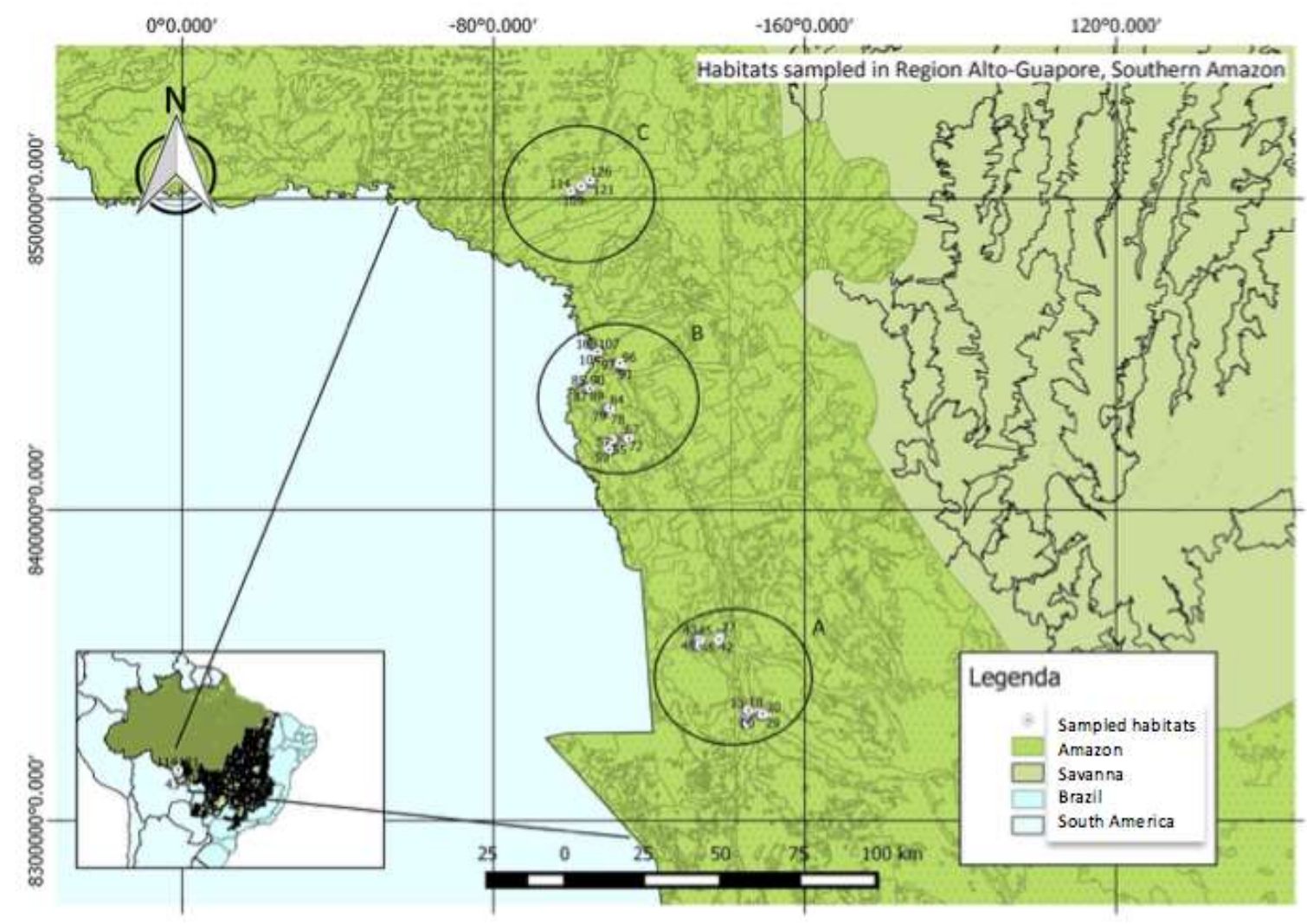

Figure1. Habitats sampled in three locations (A, B, C) in Alto-Guapore region, Southern Amazon.

We sampled three types of local habitats: marsh palm, dry forest, and flooded forest. The marsh palms are habitats of planes in Southern Amazon with riparian areas dominated by Mauritia flexuosa, flooded during the rainy period and permanently humid in the dry periods (n: 18 points). In the dry forest there is no rainfall, the height of trees fluctuates between 30 and $60 \mathrm{~m}$, with closed canopy and thick bush (n: 72 points). Flooded habitats occur along the banks of the rivers Alegre, Verde, and Guaporé. These habitats are always flooded and the trees present a maximum height of $20 \mathrm{~m}$ with lianas and aquatic plants, and without sub-woods (n: 36 points). The census procedures included the most efficient hearing census ${ }^{(11)}$, and observational census with the utilization of binoculars and photographic equipment. Identification of each foraging bird species was accomplished with the collected photographs and recordings. We also referred to specific bibliography to associate the diet of each bird species with the food available in each sampled habitat ${ }^{(11-18)}$, so that we could make inferences on each foraged habitat per bird species. We used the quantitative lifting method (adapted from Blondel et al.) ${ }^{(19)}$. We sampled points in transects equidistant $5 \mathrm{~km}$ from each other. Each transect had six points located $200 \mathrm{~m}$ apart (this distance was adequate to avoid juxtaposition of territory between most species and allowed us to cover all habitats). In total, 126 points were sampled. The recordings in each point were done using audio digital equipment (96KHz), for 15 minutes during the morning (period of the day when birds are most active), three times in different days. The sampling effort consisted of 63 days of census and 31 hours and 50 minutes of recordings covering approximately $700 \mathrm{~km}$ in length, in total. The vocalizations were then edited and the species identified, and finally revised by a bird specialist (records were deposited in the Laboratory of Mammalogy, UNEMAT, Brazil).

To investigate the presence of clustering of species relative to the type of sampled habitat, the PCA 
method was applied (Principal Components Analysis is a statistical procedure that uses an orthogonal transformation to convert a set of observations of possibly correlated variables into a set of values of linearly uncorrelated variables called principal components). We calculated the global $\beta$ diversity to measure diversity between the sampled habitats ${ }^{(2)}$. The Index quality of Jaccard (cc), a matrix of presence and absence for a dataset, represents the macrostructure of species distributed between the habitats, although application is restricted to habitats with more than seven species. This Index was calculated with the following formula:

$\mathrm{cc}=\mathrm{c} /(\mathrm{a}+\mathrm{b}+\mathrm{c})^{*} 100^{(1)}$, or

$\mathrm{cc}=\mathrm{c} /(\mathrm{A}+\mathrm{B}-\mathrm{c})^{*} 100^{(2)}$

where: "a" is the number of species unique to sample 1 , "b" is the number of specie unique to sample 2 , "c" is the number of species common to samples 1 and 2 , " $A$ " is the total number of species in sample 1 , and " $\mathrm{B}$ " is the total number of species in sample 2.

The distance values are plotted as similarity dendograms, the lower the index the less the similarity, created through the UPGMA (Unweighted Pair Group Method with Arithmetic Mean). The correlation between two matrices of dissimilarity was evaluated: one matrix of spatial distance (Euclidean) and the other one of species dissimilarity (Bray-Curtis) utilized the Mantel Test (Number of permutatitons: 999) (20). All analyzes were performed using R version 2.14.0.2011 program $^{(21)}$.

\section{Results}

A total of 148 species of birds (order Passeriformes) were identified and distributed in 27 families. Of these, $97 \%(n=145)$ of the species foraged in flooded forests, $77 \%(n=114)$ in dry forests, and $18.91 \%(n=28)$ in marsh palm. Only $18 \%(n=27)$ of the bird species foraged in all three habitats. The habitat with the highest species richness was the flooded forest ${ }^{(7)}$ (Table 1). The value of species richness per habitat and sampling areas can be seen in Table 2. The ordination showed that most species are highly associated with a habitat, for instance, Mimus saturninus is mainly observed within the flooded forest habitat, and Empidonomus varius, Onychorhynchus coronatus, Thamnomanes caesius, Myrmotherula menetriesii, and Dysithamnus mentalis in the dry forests (Figure 2). The analysis of the global beta diversities in total showed that substitution of species is predominant (Whittaker: 7.7405, Harrison: 0.14334, Cody: 554.5, Routledge: 0.700, WilsonShmida: 32.97, Mourelle: 0.610, Harrison2: 0.024, Williams: 0.5714). These values indicate that beta diversity is high. As expected, we found the same pattern when applying the measure of pairwise dissimilarity. In the cluster analysis (Figure 3), the effect of spatial distance is clear because in groups 1 and 2 the habitats closer to each other are very similar (according to the Jaccard similarity index; $r=0.872, p=0,002$ ). This is not observed with the points of groups 3 and 4 ; these points corresponded to the moment of transition and substitution of species. However, in the majority of cases, the influence of spatial distance was confirmed with the partial Mantel analysis. The influence of spatial distance on the dissimilarity index (beta diversity) was significant (Mantel statistic r: 0.0608, p (value): 0.0049) (Figure 4). 
Table I. Composition of the list of birds (order Passenformes) in Alto-Guapore region, Southem Amazon 2012

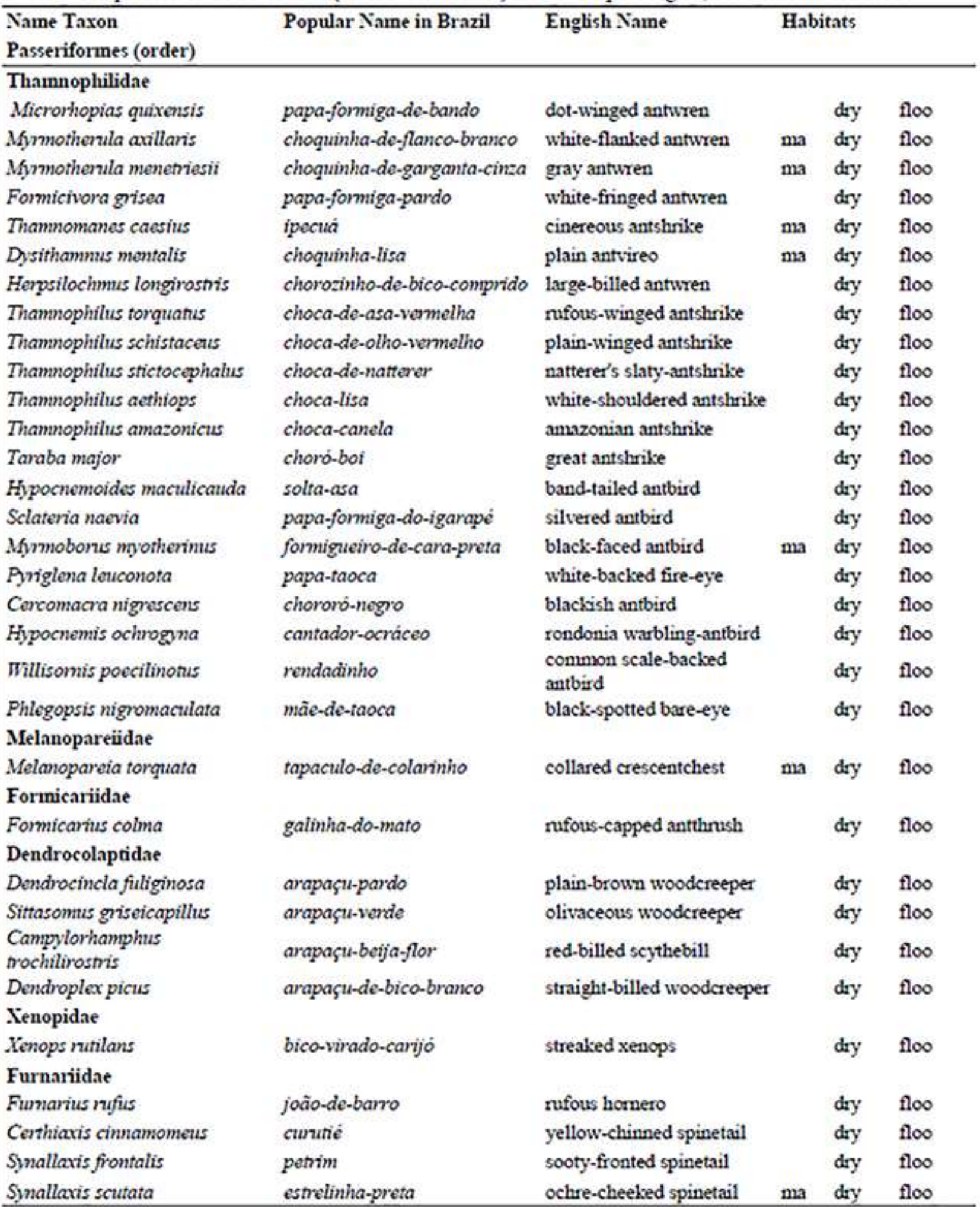

To be continued... 
Continuation

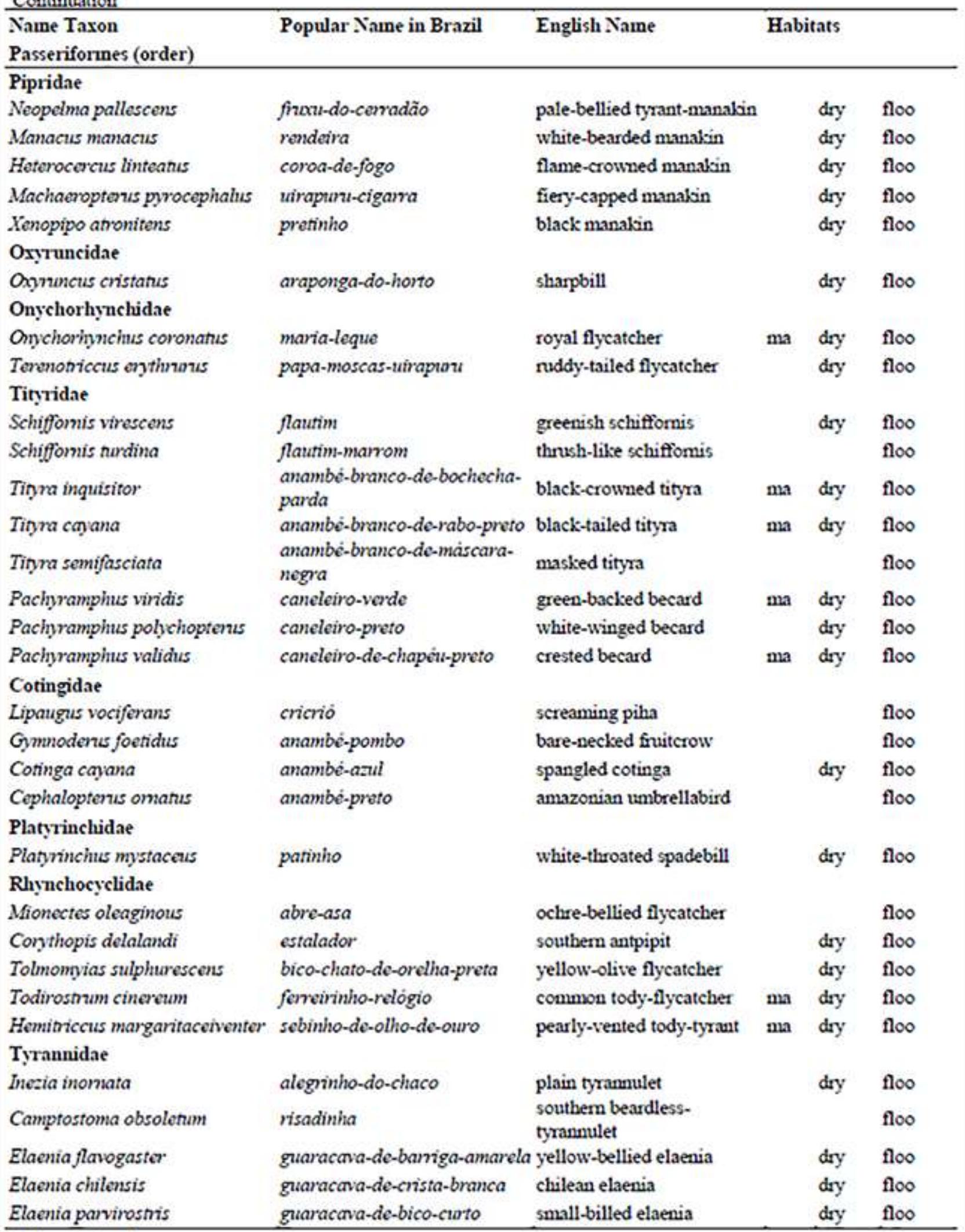

To be continued... 
Continuation

\begin{tabular}{|c|c|c|c|c|c|}
\hline \multirow{2}{*}{$\begin{array}{l}\text { Name Taxon } \\
\text { Passeriformes (order) }\end{array}$} & \multirow{2}{*}{ Popular Name in Brazil } & \multirow[t]{2}{*}{ English Name } & \multicolumn{3}{|c|}{ Habitats } \\
\hline & & & & & \\
\hline Elaenia chiriquensis & chibum & lesser elaenia & & dry & floo \\
\hline Myiopagis caniceps & guaracava-cinzenta & gray elaenia & & dry & floo \\
\hline Atrila cinnamomeus & tinguaçu-femugem & cinnamon attila & & dry & floo \\
\hline Atrila bolivianus & bate-para & dull-capped attila & & & floo \\
\hline Attila spadicens & capitão-de-saira-amarelo & bright-rumped attila & & & floo \\
\hline Ramphotrigon ruficauda & bico-chato-de-rabo-vermelho & rufous-tailed flatbill & & dry & floo \\
\hline Myiarchus swainsoni & $i m \dot{e}$ & swainson's flycatcher & & dry & floo \\
\hline Sirystes sibilator & gritador & sirystes & & & floo \\
\hline Casiomis nufis & maria-fenugem & rufous casiornis & & dry & floo \\
\hline Pitangus sulphuratus & bem-te-vi & great kiskadee & & & floo \\
\hline Philohydor lector & bentevizinho-do-brejo & lesser kiskadee & & dry & floo \\
\hline Machetomis rixosa & suinin-cavaleiro & cattle tyrant & & & floo \\
\hline Tynamnopsis sulphurea & suinin-de-garganta-rajada & sulpluxy flycatcher & & & floo \\
\hline Myiosetetes cayanensis & bentevizinho-de-asa-fenugined & rusty-margined flycatcher & & dry & floo \\
\hline Tyrannus albogulanis & suinin-de-garganta-branca & white-throated kingbird & $\mathrm{ma}$ & dry & floo \\
\hline Tyrannus savanna & tesourinha & fork-tailed flycatcher & $\mathrm{ma}$ & dry & floo \\
\hline $\begin{array}{l}\text { Griseonrannus } \\
\text { aurantioatrocristanis }\end{array}$ & peitica-de-chapét-preto & crowned slaty flycatcher & & dry & floo \\
\hline Empidonomus varius & peitica & variegated flycatcher & & dry & floo \\
\hline Fyrocephahs nubinus & principe & vermilion flycatcher & & dry & floo \\
\hline Anmdinicola leucocephala & freininha & white-headed marsh tyrant & & dry & floo \\
\hline Lathrotriccus euleri & enfenujado & euler's flycatcher & & dry & floo \\
\hline \multicolumn{6}{|l|}{ Vireonidae } \\
\hline Vireo olivaceus & junniara-boreal & red-eyed vireo & $\mathrm{ma}$ & dry & floo \\
\hline \multicolumn{6}{|l|}{ Corvidae } \\
\hline Cyanocorax cyanomelas & gralha-do-pantanal & purplish jay & & dry & floo \\
\hline Cyanocorax chrysops & gralha-picaşa & plush-crested jay & $\mathrm{ma}$ & dry & floo \\
\hline \multicolumn{6}{|l|}{ Hirundinidae } \\
\hline Stelgidopteryx nuficollis & andorinha-serradora & $\begin{array}{l}\text { soutbern rough-winged } \\
\text { swallow }\end{array}$ & & & floo \\
\hline Progne tapera & andorinha-do-campo & brown-chested martin & & & floo \\
\hline Progne chalybea & andorinha-doméstica-grande & gray-breasted martin & & dry & floo \\
\hline Tachycineta albiventer & andorinha-do-rio & white-winged swallow & & dry & floo \\
\hline Riparia niparia & andorinha-do-barnanco & bank: swallow & $\mathrm{ma}$ & dry & floo \\
\hline \multicolumn{6}{|l|}{ Troglodytidae } \\
\hline Troglodytes musculus & comuira & southern house wren & & dry & floo \\
\hline Campylorhynchus rurdimus & catatau & thrush-like wren & & dry & floo \\
\hline Phougopedius genibarbis & ganinchão-pai-avö & moustached wren & & & floo \\
\hline Cantorchilus leucotis & $\begin{array}{l}\text { garninchão-de-barniga- } \\
\text { vermelha }\end{array}$ & buff-breasted wren & & dry & floo \\
\hline Cantorchilus guarayanus & ganincha-do-oeste & fawn-breasted wren & & dry & floo \\
\hline \multicolumn{6}{|l|}{ Donacobiidae } \\
\hline Donacobius atricapilla & japacanim & black-capped donacobius & & & floo \\
\hline
\end{tabular}

To be continued... 
Continuation

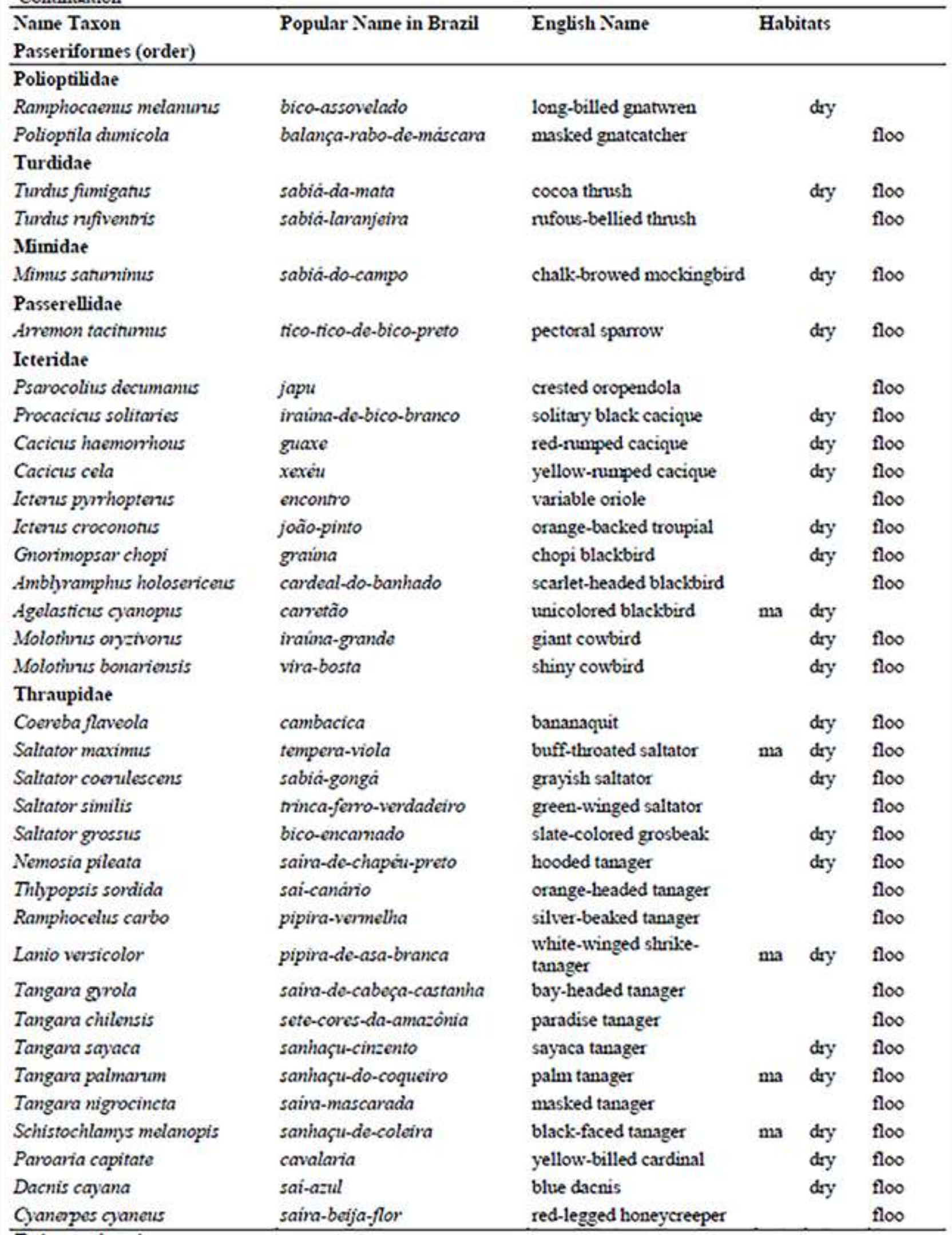


Continuation

\begin{tabular}{|c|c|c|c|c|c|}
\hline $\begin{array}{l}\text { Name Taxon } \\
\text { Passeriformes (order) }\end{array}$ & Popular Name in Brazil & English Name & \multicolumn{2}{|c|}{ Habitats } & \multirow[b]{2}{*}{ floc } \\
\hline Chlorophanes spiza & sai-verde & green honeycreeper & & & \\
\hline Hemithraupis flavicollis & saina-galega & yellow-backed tanager & & dry & floo \\
\hline Conirostram speciosum & figuinha-de-rabo-castanho & chestnut-vented conebill & & dry & floo \\
\hline Sicalis flaveola & canánio-da-terra-verdadeiro & saffron finch & & dry & \\
\hline Volatinia jacanina & tiziu & blue-black grassquit & $\mathrm{ma}$ & dry & floo \\
\hline Sporophila collaris & coleiro-do-brejo & rusty-collared seedeater & & dry & floo \\
\hline Sporophila lineola & bigodinho & lined seedeater & & & floo \\
\hline Sporophila nigricollis & baiano & yellow-bellied seedeater & & & floo \\
\hline Sporophila boinreuil & caboclinho & cooper seedeater & & & floo \\
\hline Sporophila angolensis & curio & chestnut-bellied seed-finch & & dry & floo \\
\hline \multicolumn{6}{|l|}{ Cardinalidae } \\
\hline Piranga flava & sanhaçu-de-fogo & hepatic tanager & & & floo \\
\hline Phencticus aurooventis & rei-do-bosque & black-backed grosbeak & $\mathrm{ma}$ & dry & floo \\
\hline \multicolumn{6}{|l|}{ Fringillidae } \\
\hline Euphonia chlorotica & fim-fim & purple-throated euphonia & $\mathrm{ma}$ & dry & floo \\
\hline Euphonia violacea & gaturamo-verdadeiro & violaceous euphonia & & dry & floo \\
\hline Family & 27 & & & & \\
\hline Species & 148 & & & & \\
\hline
\end{tabular}


Table II. Characteristics of the sampling areas. Latitude and longitude are shown in UTM

\begin{tabular}{|c|c|c|c|c|c|}
\hline $\begin{array}{l}\text { Code } \\
\text { map }\end{array}$ & Habitat & Areas & Latitude & Longitude & $\begin{array}{l}\text { Passeriformes } \\
\text { richness }\end{array}$ \\
\hline 1 & Flooded forest & $\mathrm{A}$ & 180925 & 8331295 & 42 \\
\hline 2 & Flooded forest & A & 181142 & 8331279 & 41 \\
\hline 3 & Flooded forest & A & 181372 & 8331224 & 50 \\
\hline 4 & Flooded forest & A & 181021 & 8331896.89 & 20 \\
\hline 5 & Dry forest & A & 181299 & $\$ 331786$ & 24 \\
\hline 6 & Dry forest & A & 181531 & 8331695 & 1 \\
\hline 7 & Flooded forest & A & 181298 & 8333552 & 63 \\
\hline 8 & Flooded forest & A & 181210 & 8333807 & 41 \\
\hline 9 & Flooded forest & A & 181133 & $\$ 334009$ & 48 \\
\hline 10 & Flooded forest & A & 180970 & 8334329 & 40 \\
\hline 11 & Dry forest & A & 180869 & 8334506 & 34 \\
\hline 12 & Dry forest & A & 180743 & 8334696 & 34 \\
\hline 13 & Flooded forest & A & 180507 & 8335421 & 30 \\
\hline 14 & Flooded forest & A & 180859 & 8335484 & 21 \\
\hline 15 & Flooded forest & A & 181109 & 8335564 & 19 \\
\hline 16 & Flooded forest & A & 181345 & 8335637 & 0 \\
\hline 17 & Flooded forest & A & 181644 & 8335637 & 1 \\
\hline 18 & Flooded forest & A & 181916 & 8335643 & 0 \\
\hline 19 & Marsh palm & A & 185524.73 & 8334058.32 & 3 \\
\hline 20 & Marsh palm & A & 185483 & $\$ 334233$ & 6 \\
\hline 21 & Marsh palm & A & 185408 & 8334423 & 9 \\
\hline 22 & Marsh palm & A & 185274 & $\$ 334561$ & 13 \\
\hline 23 & Marsh palm & A & 185131 & 8334699 & 9 \\
\hline 24 & Marsh palm & A & 184996 & 184996 & 1 \\
\hline 25 & Marsh palm & A & 186507.2 & 8333914.13 & 2 \\
\hline 26 & Marsh palm & A & 186719 & 8333932 & 9 \\
\hline 27 & Marsh palm & A & 186719 & 8333932 & 12 \\
\hline 28 & Marsh palm & A & 187144 & 8334029 & 7 \\
\hline 29 & Marsh palm & A & 187354 & 8334066 & 5 \\
\hline 30 & Marsh palm & A & 187554 & 8334110 & 2 \\
\hline 31 & Marsh palm & A & 185494.87 & 8333830.27 & 1 \\
\hline 32 & Marsh palm & A & 185700 & 8333887 & 9 \\
\hline 33 & Marsh palm & A & 185901 & 8333956 & 9 \\
\hline 34 & Marsh palm & A & 186077 & 8334058 & 8 \\
\hline 35 & Marsh palm & $\mathrm{A}$ & 186249 & $\$ 334220$ & 5 \\
\hline 36 & Marsh palm & A & 186402 & 8334340 & 2 \\
\hline 37 & Dry forest & A & $\$ 19055.33$ & 8359824.1 & 14 \\
\hline 38 & Dry forest & A & 818975 & 8359651 & 9 \\
\hline 39 & Dry forest & A & $\$ 1 \$ 817$ & 8359468 & 4 \\
\hline 40 & Dry forest & A & $\$ 1 \$ 689$ & $\$ 359326$ & 5 \\
\hline
\end{tabular}

To be continued... 
Continuation...

\begin{tabular}{|c|c|c|c|c|c|}
\hline $\begin{array}{l}\text { Code } \\
\text { map }\end{array}$ & Habitat & Areas & Latitude & Longitude & $\begin{array}{l}\text { Passeriformes } \\
\text { richness }\end{array}$ \\
\hline 41 & Dry forest & $\mathrm{A}$ & 818493 & 8359114 & 7 \\
\hline 42 & Dry forest & A & $\$ 18255$ & 8358369 & 8 \\
\hline 43 & Dry forest & A & $\$ 11167.56$ & 8359219.72 & 9 \\
\hline 44 & Dry forest & A & $\$ 11318$ & 8359025 & 9 \\
\hline 45 & Dry forest & A & $\$ 11433$ & 8358841 & 6 \\
\hline 46 & Dry forest & A & $\$ 11602$ & 8358642 & 2 \\
\hline 47 & Dry forest & A & 811854 & 8358500 & 2 \\
\hline 48 & Dry forest & A & 812095 & 8358369 & 5 \\
\hline 49 & Dry forest & A & 810624.6 & 8356568.87 & 8 \\
\hline 50 & Dry forest & A & 810867 & 8356679 & 7 \\
\hline 51 & Dry forest & A & 811116 & 8356721 & 1 \\
\hline 52 & Dry forest & A & $\$ 11397$ & 8356733 & 1 \\
\hline 53 & Flooded forest & A & $\$ 11692$ & 8356753 & 4 \\
\hline 54 & Flooded forest & A & $\$ 11981$ & 8356764 & 3 \\
\hline 55 & Dry forest & B & 785583 & $\$ 420492$ & 13 \\
\hline 56 & Dry forest & B & 785329 & $\$ 420504$ & 4 \\
\hline 57 & Dry forest & B & 785110 & 8420518 & 1 \\
\hline 58 & Dry forest & B & 784857 & 8420483 & 3 \\
\hline 59 & Dry forest & B & 784609 & $\$ 420485$ & 3 \\
\hline 60 & Dry forest & B & 784418 & 8420524 & 2 \\
\hline 61 & Dry forest & B & 786562.51 & 8423589.41 & 6 \\
\hline 62 & Dry forest & B & 786356 & 8423564 & 1 \\
\hline 63 & Dry forest & B & 786159 & 8423565 & 2 \\
\hline 64 & Dry forest & B & 785941 & $\$ 423609$ & 5 \\
\hline 65 & Dry forest & B & 785736 & 8423655 & 4 \\
\hline 66 & Dry forest & B & 785535 & 8423743 & 3 \\
\hline 67 & Dry forest & B & 789791.98 & 8424305.24 & 3 \\
\hline 68 & Dry forest & B & 789985 & $\$ 424198$ & 0 \\
\hline 69 & Dry forest & B & 790212 & $\$ 424129$ & 3 \\
\hline 70 & Dry forest & B & 790422 & 8424038 & 2 \\
\hline 71 & Dry forest & B & 790681 & $\$ 423946$ & 5 \\
\hline 72 & Dry forest & B & 790951 & $\$ 423856$ & 3 \\
\hline 73 & Dry forest & B & 784229.14 & 8432586.27 & 2 \\
\hline 74 & Dry forest & B & 784437 & 8432581 & 4 \\
\hline 75 & Dry forest & B & 784660 & $\$ 432583$ & 1 \\
\hline 76 & Dry forest & B & 784888 & $\$ 432615$ & 3 \\
\hline 77 & Dry forest & B & 785113 & 8432621 & 1 \\
\hline 78 & Dry forest & B & 785331 & 8432631 & 1 \\
\hline 79 & Dry forest & B & 784048.69 & 8433618.56 & 2 \\
\hline 80 & Dry forest & B & 784208 & 8433773 & 4 \\
\hline 81 & Dry forest & B & 784428 & $8433 \$ 25$ & 2 \\
\hline
\end{tabular}

To be continued... 
Continuation

\begin{tabular}{|c|c|c|c|c|c|}
\hline $\begin{array}{l}\text { Code } \\
\text { map }\end{array}$ & Habitat & Areas & Latitude & Longitude & $\begin{array}{l}\text { Passeriformes } \\
\text { richness }\end{array}$ \\
\hline 82 & Dry forest & B & 784658 & 8433875 & 0 \\
\hline 83 & Dry forest & B & 784878 & 8433895 & 2 \\
\hline 84 & Dry forest & B & 785122 & $\$ 433926$ & 0 \\
\hline 85 & Dry forest & B & 777628.65 & 8440405.58 & 3 \\
\hline 86 & Dry forest & B & 777927 & $\$ 440412$ & 4 \\
\hline 87 & Dry forest & B & 778204 & $\$ 440352$ & 3 \\
\hline 88 & Dry forest & B & 778535 & 8440404 & 2 \\
\hline 89 & Dry forest & B & 778829 & 8440401 & 1 \\
\hline 90 & Dry forest & B & 779083 & 8440399 & 2 \\
\hline 91 & Flooded forest & B & 788291 & 8447170 & 10 \\
\hline 92 & Flooded forest & B & 788500 & 8447253 & 1 \\
\hline 93 & Flooded forest & B & 788723 & $\$ 447334$ & 4 \\
\hline 94 & Flooded forest & B & 788940 & $\$ 447398$ & 7 \\
\hline 95 & Flooded forest & B & 789158 & $\$ 447458$ & 2 \\
\hline 96 & Dry forest & B & 789402 & $\$ 447510$ & 5 \\
\hline 97 & Flooded forest & B & 787388.85 & $\$ 4476 \$ 6.02$ & 12 \\
\hline 98 & Flooded forest & B & 787651 & $\$ 447772$ & 6 \\
\hline 99 & Flooded forest & B & 787875 & $\$ 447841$ & 3 \\
\hline 100 & Flooded forest & B & 788140 & $\$ 447970$ & 6 \\
\hline 101 & Flooded forest & B & 788443 & 8448085 & 3 \\
\hline 102 & Dry forest & B & 788652 & $\$ 44 \$ 150$ & 1 \\
\hline 103 & Flooded forest & B & 782787.56 & $\$ 451826.06$ & 11 \\
\hline 104 & Flooded forest & B & 782582 & 8451806 & 8 \\
\hline 105 & Flooded forest & B & 782381 & 8451887 & 6 \\
\hline 106 & Flooded forest & B & 782079 & 8451917 & 15 \\
\hline 107 & Flooded forest & B & 781864 & 8451969 & 3 \\
\hline 108 & Dry forest & B & 781666 & 8452017 & 3 \\
\hline 109 & Flooded forest & $\mathrm{C}$ & 775268 & 8503059 & 4 \\
\hline 110 & Flooded forest & $\mathrm{C}$ & 775018 & 8503202 & 9 \\
\hline 111 & Flooded forest & $\mathrm{C}$ & 774840 & 8503339 & 8 \\
\hline 112 & Flooded forest & $\mathrm{C}$ & 774682 & $\$ 503490$ & 18 \\
\hline 113 & Flooded forest & $\mathrm{C}$ & 774517 & 8503621 & 15 \\
\hline 114 & Dry forest & $\mathrm{C}$ & 774349 & $\$ 503779$ & 3 \\
\hline 115 & Dry forest & $\mathrm{C}$ & 778523.36 & 8504561.8 & 1 \\
\hline 116 & Dry forest & $\mathrm{C}$ & 778807 & $85043 \$ 1$ & 17 \\
\hline 117 & Dry forest & $\mathrm{C}$ & 778328 & $\$ 50467 \mathrm{~S}$ & 18 \\
\hline 118 & Dry forest & $\mathrm{C}$ & 778178 & $\$ 504870$ & 15 \\
\hline 119 & Dry forest & $\mathrm{C}$ & 777995 & 8505131 & 13 \\
\hline 120 & Dry forest & $\mathrm{C}$ & 777804 & 8505374 & 1 \\
\hline 121 & Dry forest & $\mathrm{C}$ & 781622.42 & 8506496.55 & 1 \\
\hline 122 & Dry forest & C & 781296 & 8506654 & 13 \\
\hline
\end{tabular}

To be continued 
Continuation

\begin{tabular}{llllll}
\hline $\begin{array}{l}\text { Code } \\
\text { map }\end{array}$ & Habitat & Areas & Latitude & Longitude & $\begin{array}{l}\text { Passeriformes } \\
\text { richness }\end{array}$ \\
\hline 123 & Dry forest & C & 781175 & $\$ 506925$ & 11 \\
124 & Dry forest & C & 781065 & 8507070 & 3 \\
125 & Dry forest & C & 780930 & 8507258 & 13 \\
126 & Dry forest & C & 780708 & 8507364 & 1 \\
\hline
\end{tabular}

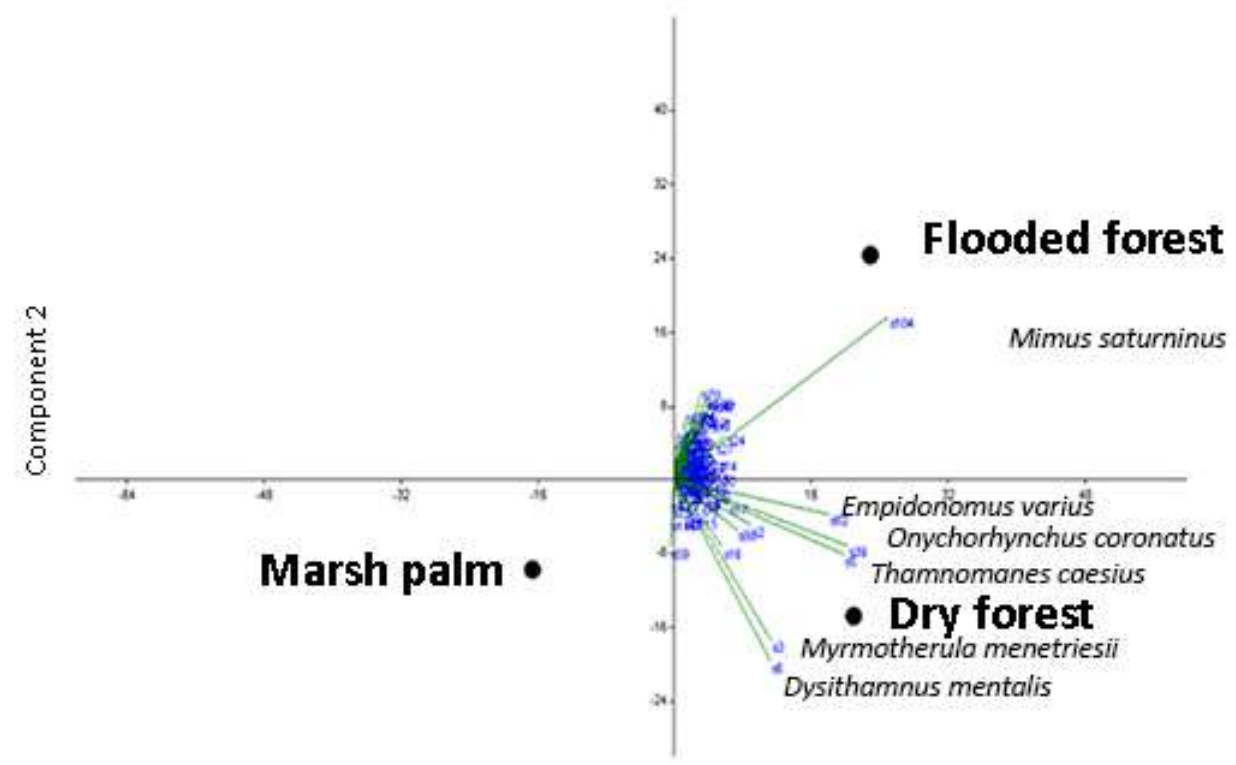

Component 1

Figure2. PCA-ordination diagram in correlation biplot scaling with birds species (Passeriformes) represented by arrows and habitats by points for data of birds from Southern Amazon, Brazil.

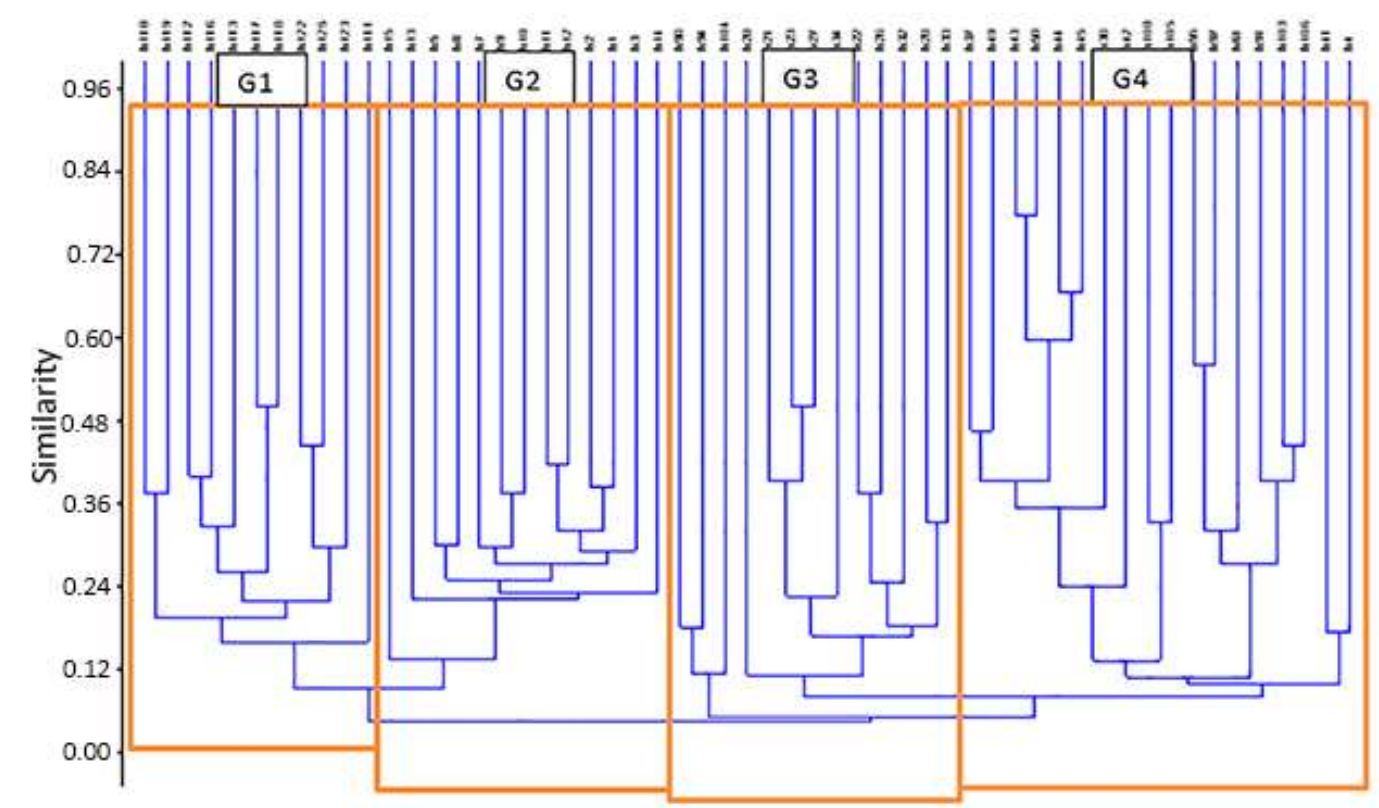

Figure3. Dendogram of index similarity Jaccard (birds order Passeriformes) for habitats ( $r=0.872$, $\mathrm{p}=0.002$ ). Acronyms: $\mathrm{h}=$ habitats, see type of habitat according table $I$ and $\mathrm{G}=$ groups principals. 


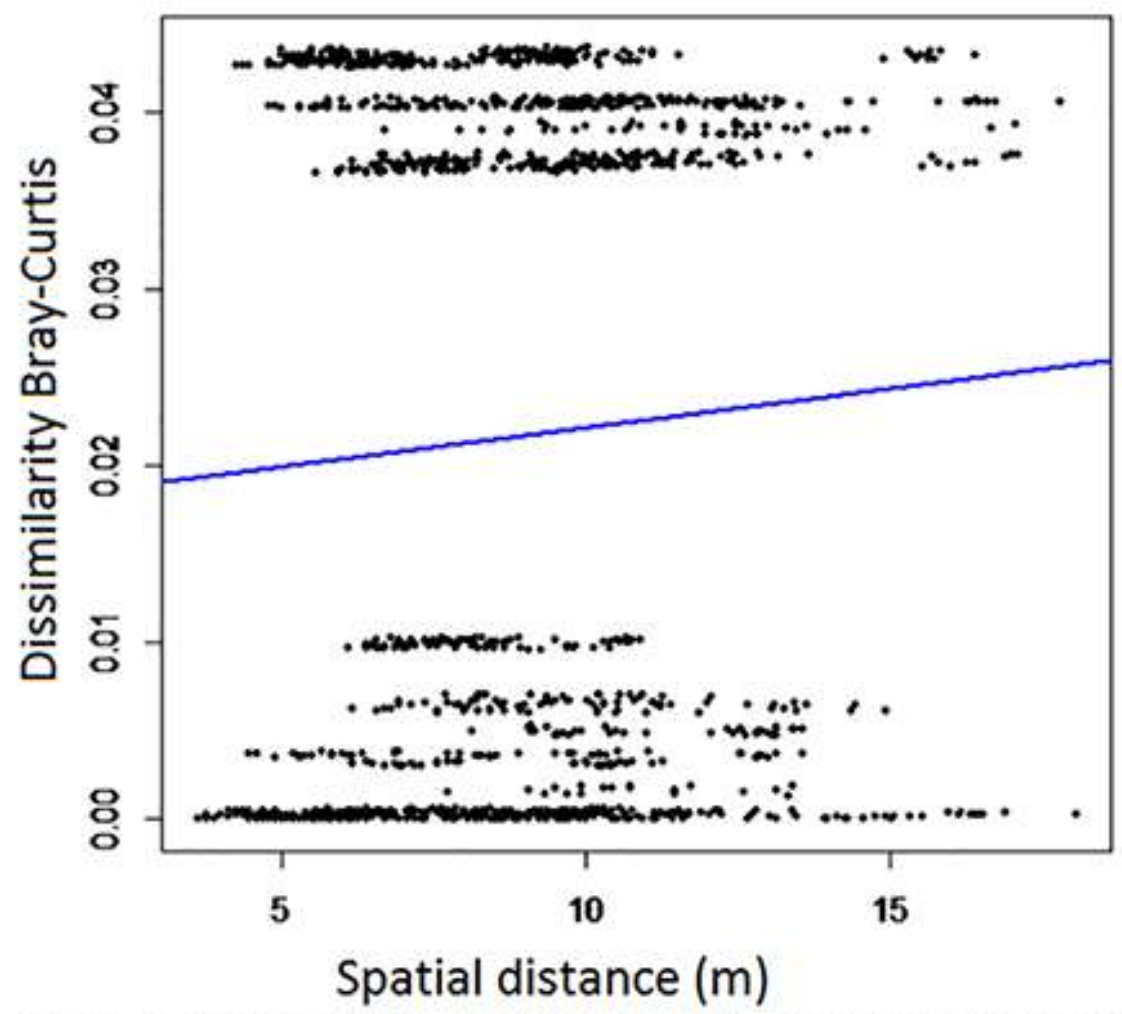

Figure 4. Mantel statistic based on Pearson's product-moment correlation, relation between species dissimilarity and spatial distance in meters of birds (order Passeriformes) in Southern Amazon ( $r: 0.0608, p$ : 0.005 ). Each point represents one pair of compared habitats.

\section{Discussion}

Silva ${ }^{(22)}$ suggested that many amazon species penetrate the Brazilian savanna biome following the gallery forest or vice versa, both places presenting a high diversity of birds ${ }^{(23)}$. In this study, the sampled places are very close to the transition between the Amazon and the Brazilian savanna, and this fact may explain the substitution of species that we observe. The high variability in the composition of species (unequally distributed) in the sampled points also contributed to the high species richness of birds Passeriformes. As suggested by Williams ${ }^{(24)}$, the increased level of environmental heterogeneity in the sampled habitats may be very relevant in explaining the high species richness that we found. Cohn-Haft, Whitaker, and Stouffer ${ }^{(25)}$ also proposed that habitat heterogeneity is the most important determinant for bird species richness inside the Amazon, and not primary productivity or level of rainfall as advocated by other authors.

Concerning the preference for habitats, this study showed that, although some species revealed to be habitat specialists, most bird species of the order Passeriformes explored and foraged many types of habitats. The habitat that presented more species was the flooded forest (Table 2). In this type of habitat, birds may find suitable conditions that directly influence their life cycle, such as, water, food, shelter, and protection from predators ${ }^{(26,27)}$. In the Amazon, many species, even residents, disperse according to water and food resources and these are abundantly present in the flooded forest habitat. Ferreira et al. ${ }^{(28)}$ found more richness of species at flooded habitats, a finding that agrees with our own results. The species Mimus saturninus had the highest level of association with 
this type of habitat, though always with open grasslands with scattered trees and shrubs close-by. We believe this may be explained by the fact that this species lives in groups of thirteen individuals and they forage the river for reproduction and food (fruits). In the dry forest habitat there are many winged insects, which could explain the presence of Empidonomus varius, Dysithamnus mentalis, Onychorhynchus coronatus, Thamnomanes caesius, and Mymotherula menetriesii in this habitat, since they primarily feed in these insects.

The high beta diversity that we found in this study is very important, particularly for strategic conservation plans. We provide specific points along an environmental gradient in the Amazon forest, where a high richness and high variation in species composition within different types of habitats may be found. More attention should be given to the importance of local conservation. High beta diversity should be taken into consideration when designing a natural reserve, particularly in including, placing and in the extension of contiguous heterogeneous habitats ${ }^{(29)}$, which contrast with lowland habitats where species are widely distributed ${ }^{(11,30,31)}$. The positive correlation between the species dissimilarity and geographic distance observed for Passeriformes in this study is in line with the Neutral Theory ${ }^{(32)}$, which states that species similarity in a community decreases with increased geographic distance between different environments. This means that because of the limitation in species distribution, the more distant the habitats are, the more different they will be in terms of species composition ${ }^{(33)}$. This author assumes that the limitation in species distribution is a function of immigration and local extinction, with species appearing and disappearing. Therefore, for species that have a small distribution, local and regional variation will impact their distribution the most. The analysis of the Jaccard similarity index revealed a major point where substitution of species is significant. Groups 3 and 4 appear on a point of transition and mixture of species close to the North Amazon, where in a small space, many different species may be found. This species composition is organized in limited local populations with high beta diversity that should be protected from the deforestation, selective logging, and poaching that have been occurring because this community is particularly vulnerable to disruption, and immediate recovery will most likely lead to reorganization of an unbalanced ecosystem. Thus, this study provides important information on specific habitats with high beta diversity whose stability is at risk in the face of continuing environmental challenges.

\section{Acknowledgements}

To the BioNorte Project - Knowledge, Sustainable Use and Bioprospecting of Biodiversity in the Southern Amazon for funding the project and to CAPES (Coordination for the Improvement of Higher Education Personnel) for the scholarship granted.

\section{References}

1. Orians GH. The number of bird species in some tropical forests. Ecology. 1969, 50: (5) 783-801. http://dx.doi.org/10.2307/1933692

2. Whittaker RH. Communities and Ecosystems. Macmillan, New York. 1975, 583p. English.

3. Koleff P, Gaston KJ, Lennon JJ. Measuring beta diversity for presence absence data. Journal of Animal Ecology. 2003, 72: (3) 367-382. http://dx.doi.org/10.1046/j.1365-2656.2003.00710.x 
4. Blair RB. Birds and butterflies along an urban gradients: Surrogate taxa for assessing biodiversity? Ecological Applications. 1999, 9: (1) 164-170. http://www.jstor.org/stable/2641176

5. Cracraft J. "Historical Biogeography and Patterns of Differentiation within the South American Avifauna: Areas of Endemism". Ornithological Monographs. 1985, 36: 49-41. http://dx.doi.org/10.2307/40168278

6. Magurran AE. Measuring biological diversity. Oxford: Blackwell Publishing. ISBN 0-632-05633-9. 2004, 2064p. English.

7. Young BE, DeRosier D, Powell GVN. Diversity and conservation of understory birds in the Tilarán mountains, Costa Rica. Auk. 1998, 115: (4) 998-1016. http://dx.doi.org/10.2307/4089518

8. Blake JG, Loiselle BA. Diversity of birds along an elevational gradient in the Cordillera Central, Costa Rica. Auk. 2000, 117: 663-686. http://dx.doi.org/10.1642/0004-8038(2000)117[0663:DOBAAE]2.0.CO;2

9. Kitayama K. An altitudinal transect study of the vegetation of Mount Kinabalú, Borneo. Vegetatio. 1992, 102: (2) 149-171. http://dx.doi.org/10.1007/bf00044731

10. Aleixo A. Lacunas de conhecimento, prioridades de pesquisa e perspectivas futuras na conservação de aves na Amazônia Brasileira. 2009, 54p. In: De Luca A, Develey PF, Bencke GA, Goerck JM. (Orgs.). Áreas importantes para a conservação das aves no Brasil: parte II - Amazônia, Cerrado e Pantanal. São Paulo: SAVE Brasil. 361p.

11. Terborgh J, Robinson SK, Parker III TA, Munn CA, Pierpont N. Structure and organization of an Amazonn forest bird community. Ecological Monographs. 1990, 60: (2) 213-238. http://dx.doi.org/10.2307/1943045

12. Moojen J, Carvalho JC, Lopes HS. Observações sobre o conteúdo gástrico das aves brasileiras. Rio de Janeiro, Memórias do Instituto Oswvaldo Cruz. 1941, 36 (3): 405-444. http://dx.doi.org/10.1590/S0074$\underline{02761941000300016}$

13. Hempel A. Estudo da alimentação natural de aves silvestres do Brasil. São Paulo, Arquivos do Instituto Biológico. 1949, 19: 237-268. http://www.scielo.br/scielo.php?script=sci_nlinks\&ref=000042\&pid=S0071$\underline{1276195100010002200009 \& \operatorname{lng}=\mathrm{en}}$

14. Kuhlmann M, Jimbo S. A flora na alimentação das aves brasileira: I Generalidades. São Paulo, Papéis $\begin{array}{lllll}\text { Avulsos de } & \text { Zoologia. } & 1957, & 13(7): & \text { 85-97. }\end{array}$ http://www.scielo.br/scielo.php?script=sci_nlinks\&ref=7299265\&pid=S0101$8175200500040002400028 \& \operatorname{lng}=$ en

15. Schubart O, Aguirre AC, Sick H. Contribuição para o conhecimento da alimentação das aves brasileiras.

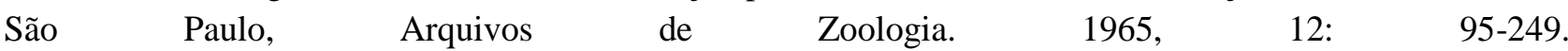
http://www.scielo.br/scielo.php?script=sci_nlinks\&ref=7299277\&pid=S0101$\underline{8175200500040002400040 \& \operatorname{lng}=\mathrm{en}}$

16. Willis EO. The compositions of avian communities in remanescents woodlots in southern Brazil. Papéis $\begin{array}{lllll}\text { Avulsos do } & \text { Museu } & \text { Zoologia. } & \text { 1979, } & \text { 33:1-25. }\end{array}$ http://www.scielo.br/scielo.php?script=sci_nlinks\&ref=7357770\&pid=S0101$\underline{8175200700020001500025 \& \operatorname{lng}=\text { en }}$

17. Motta-Junior JC. Estrutura trófica e composição das avifaunas de três habitats terrestres na região central do Estado de São Paulo. Ararajuba. 1990, 1:65-71.

18. Sick H. Ornitologia brasileira. Rio de Janeiro, Nova Fronteira, II+862. SIMBERLOFF, D. 1997, 862p.

19. Blondel J, Ferry C, Frochot B. Point counts with unlimited distance. Stud. Avian Biol. 1981, 6: 414-420.

20. Mantel N. The detection of disease clustering and a generalized regression approach. Cancer Research. 
1967, 27: 209-220.

21. R Development Core Team. R: A language and environment for statistical computing. Vienna, $\mathrm{R}$ Foundation for Statistical Computing, ISBN 3900051-07-0. 2011. available online at: http://www.Rproject.org.

22. Silva JMC. Distribuition of Amazonn and Atlantic birds in gallery forest of the Cerrado region, South America. Ornit. Neot. 1967, 7 (1) :1-18.

23. Tocantins. Avaliação Ecológica Rápida do Parque Estadual do Cantão. Secretaria do Planejamento e Meio Ambiente do Estado do Tocantins, Palmas. 2004a.

24. Williams CB. Patterns in the balance of nature. New York: New York Academic Press. 1964, 300p. English

25. Cohn-Haft M, Whittaker A, Stouffer PC. A second look at the "species-poor" central Amazon: the avifauna north of Manaus, Brazil. 1967, 235p. in: Remsen, J.V., ed. Studies in Neotropical ornithology honoring Ted Parker. Ornithological Monograph. 1967, 48. http://dx.doi.org/10.2307/40157535

26. Parker III. Neotropical birds: ecology and conservation. Chicago, University of Chicago Press.

27. Willson MF. Avian community organization and habitat structure. Ecology. 1974, 55: (5) 1017-1027. http://dx.doi.org/10.2307/1940352

28. Ferreira LV, Almeida SS, Amaral DD, Parolin P. Riqueza e Composição de Espécies da Floresta de Igapó e Várzea da Estação Científica Ferreira Pena: Subsídios para o plano de Manejo da Floresta Nacional de Caxianã. 2005, [cited 2016 Jun 7]. Available from: http://www.anchietano.unisinos.br/publicacoes/botanica/botanica56.htm\#05

29. Lombard AT, Hilton-Taylor C, Rebelo AG, Pressey RL, Cowling RM. Reserve selection in the Succulent Karoo, South Africa: coping with high compositional turnover. Plant Ecology. 1999, 142: (1/2) 35-55. http://dx.doi.org/10.1023/a:1009866126729

30. Robinson WD, Brawn JD, Robinson SK. Forest Bird Community Structure In Central Panamá: Influence Of Spatial Scale And Biogeography. Ecological Monographs. 2000, 70, 209-235.

31. Condit R, Pitman N, Leigh Jr EG, Chave J, Terborgh J, Foster RB, Nuñez VP, Aguilar S, Valencia R, Villa G, Muller-Landau HC, Losos E, Hubbell SP. Beta-diversity in tropical forest trees. Science. 2002, 295, 666-669.

32. Hubbell SP. The united neutral theory of biodiversity and biogeography. Princeton University Press, Princeton. 2001, "The Unified Neutral Theory of Biodiversity and Biogeography (MPB-32)"

33. Hubbell SP. Neutral theory and the evolution of ecological equivalence. Ecology. 2006, 87: (6) 13871398. http://dx.doi.org/10.1890/0012-9658(2006)87[1387:ntateo]2.0.co;2 